\title{
Nonlinear Dynamics of the Additive-Pulse Modelocked Laser
}

\author{
E.J. MOZDY and C.R. POLLOCK* \\ School of Electrical Engineering, Phillips Hall, Cornell University, Ithaca, NY 14853, USA
}

(Received 13 January 1998)

\begin{abstract}
We have modeled the additive-pulse modelocked (APM) laser with a set of four nonlinear difference equations, that describe the transit of optical pulses through the main cavity and through an external cavity containing a single-mode optical fiber. Simulating the system under several parameter variations, including fiber length, gain, and fiber coupling, we have observed period-doubling bifurcations into chaos. In addition, the model predicted large regimes of quasiperiodicity, and crisis transitions between different chaotic regions. We have used the method of nearest neighbors, Lyapunov exponents, and attractor reconstruction to characterize the chaotic regimes and the different types of bifurcations. We have included bandwidth-limiting and monitoring provisions to prevent non-physical solutions. To our knowledge, this is the first such characterization of chaos in the APM laser, as well as the first evidence of crisis behavior.
\end{abstract}

Keywords: Modelocked, Laser, Model, Chaos, Bifurcation

\section{INTRODUCTION}

Quasiperiodicity, period-doublings, and chaos have recently been identified in the pulsed output of the additive-pulse modelocked (APM) laser [1,2], sparking a renewed interest in this system related to the study and possible exploitation of these complex nonlinearities. In this paper, we present the results of discrete mathematical simulations aimed at properly characterizing the dynamics of the APM laser through the calculation of embedding dimension and Lyapunov exponents, the analysis of unstable time series, and the reconstruction of chaotic attractors. With these efforts, we hope to provide the necessary evidence that the unstable APM output is truly deterministic, and explore more subtle issues such as the varied bifurcation parameters and routes to chaos in this laser.

APM has been used extensively since about 1984 to provide transform-limited, ultrashort optical pulses from a variety of gain media [3-8]. The most common APM laser configuration, shown in Fig. 1, consists of two, coupled cavities: the main cavity houses the gain, tuning elements, high reflector, and output coupler of the laser while the external cavity contains a short length of optical fiber, and

*Corresponding author. Tel.: 607-255-5032. 


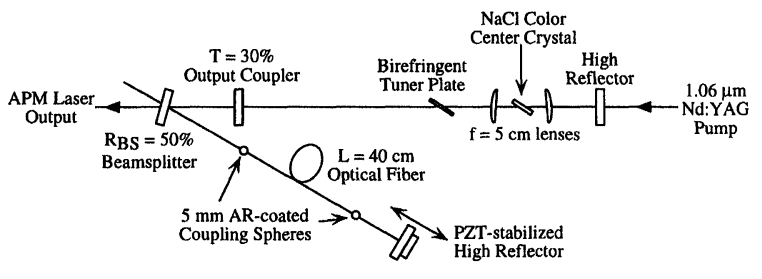

FIGURE 1 The NaCl APM laser cavity.

is exactly matched in length to the main cavity. Considered alone, the main cavity is either synchronously pumped or actively modelocked (e.g. by an acousto-optic modulator [9]) to produce relatively long $(10 \mathrm{ps})$ pulses. In the APM configuration, a fraction of the pulsed output power is coupled onto the optical fiber of the external cavity, and then retroreflected back to recombine with the pulses circulating in the main cavity. The small fiber core diameter leads to high peak intensities, causing a frequency chirp due to nonlinear self-phase modulation (SPM). When the length of the external cavity is properly adjusted, the frequency-chirped pulses interfere with those of the main cavity constructively at the peaks, and destructively at the wings, resulting in an effective temporal shortening of the pulses. After many round trips, dispersion and finite bandwidth balance the pulse shortening caused by this process, and the laser reaches a steady-state operating point, generating pulses on the order of $100 \mathrm{fs}$ in duration.

Because modelocked lasers are characterized by a (periodic) train of pulses at the output coupler separated by the main cavity round-trip transit time, such lasers are most easily described by an iterative model. In previous work [1], four difference equations were used to model the APM laser, verify steady-state operation, optimize laser parameters, and predict the presence of bifurcations and chaos. Despite these achievements, no one has pursued these equations in a more detailed understanding of the APM dynamics. In particular, do quasiperiodicity and chaos truly exist in the APM laser and if so, can the chaos be characterized by a largest Lyapunov exponent? What is the dimensionality of the chaotic system, and can one reconstruct a strange attractor from the time series output of the iterative model? What routes to chaos exist in the APM system? Although the iterative model has been used to suggest rich chaotic dynamics, these questions indicate the need for better characterization.

We have pursued these questions at length, and will describe the development and use of the iterative APM model. In determining a proper embedding dimension for the chaotic dynamics, calculating a largest Lyapunov exponent, and reconstructing several different attractors from the output time series, we provide convincing evidence that this laser's unstable output is truly deterministic. We also explore the routes to chaos, which include period-doubling and crisis transitions in different regions of phase space.

\section{THE APM MODEL}

The APM laser can be represented schematically as shown in Fig. 2. The quantities $a_{1}, a_{2}, b_{1}$, and $b_{2}$ model the incident and reflected electric fields at the output coupler. The laser pulses can be modeled by some optical carrier wave and a Gaussian envelope:

$$
E(t)=A \exp (\mathrm{i} \omega t) \exp \left(-2 \ln 2\left[t-t_{0}\right]^{2} / \delta t_{0}^{2}\right) \exp (\mathrm{i} \phi)
$$

Since the carrier wave is common to all fields in the APM model, it can be omitted and the pulses are simply represented by the complex envelope. The two cavities interact with each other at the output coupler, which has an intensity reflectivity $R$. Two difference equations explain the interaction of

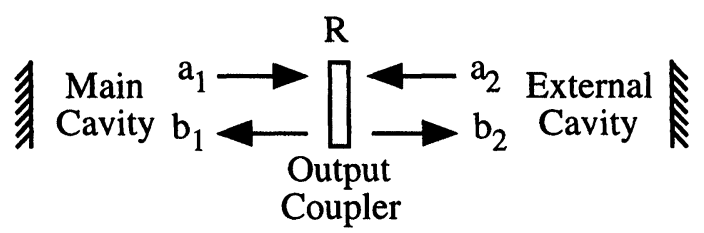

FIGURE 2 A schematic of the APM cavity, defining the model fields $a_{1}, a_{2}, b_{1}$, and $b_{2}$. 
these fields:

$$
\begin{aligned}
& b_{1}=a_{1} \sqrt{R}+a_{2} \sqrt{1-R}, \\
& b_{2}=a_{1} \sqrt{1-R}-a_{2} \sqrt{R} .
\end{aligned}
$$

The $b$ fields generated above make a round trip traverse of their respective cavities in becoming $a$ fields. Defining all of the optical processes in those traverses allows us to develop a description of the pulse evolution which can then be iterated. From Fig. 1, the field $b_{1}$ passes through the birefringent tuner plate (BTP) and saturable gain twice before returning to the output coupler as $a_{1}$. The tuner plate, positioned at Brewster's angle $\left(\sim 56.8^{\circ}\right)$ in the main cavity, has an intensity transmission that depends upon wavelength as [10]

$$
\begin{gathered}
I_{\mathrm{T}}(\lambda)=1-\sin ^{2}(2 \phi) \frac{n_{\mathrm{o}}^{4}-n_{\mathrm{o}}^{2} \cos ^{2} \theta}{\left(n_{\mathrm{o}}^{2}-\cos ^{2} \phi \cos ^{2} \theta\right)^{2}} \\
\times \sin ^{2}\left(\frac { \pi t } { \lambda } \left\{\frac{n_{\mathrm{e}}\left[1+\cos ^{2} \theta \cos ^{2} \phi\left(1 / n_{\mathrm{e}}^{2}-1 / n_{\mathrm{o}}^{2}\right)\right]}{\left[1-\cos ^{2} \theta\left(\sin ^{2} \phi / n_{\mathrm{e}}^{2}+\cos ^{2} \phi / n_{\mathrm{o}}^{2}\right)\right]^{1 / 2}}\right.\right. \\
\left.\left.-\frac{n_{\mathrm{o}}}{\left[1-\cos ^{2} \theta / n_{\mathrm{o}}^{2}\right]^{1 / 2}}\right\}\right),
\end{gathered}
$$

where $\theta$ is the angle formed by the surface of the plate and the cavity optic axis, $\phi$ is the angular deviation of the plate's extraordinary axis from the vertical, $t$ is the plate thickness, and $n_{\mathrm{o}}\left(n_{\mathrm{e}}\right)$ is the index of refraction of the (extra)ordinary axis. For this model, we used a plate of thickness $t=1.75 \mathrm{~mm}$, which is similar to that used in the laboratory.

The laser gain both amplifies the pulse and performs some spectral filtering, due to its finite gain bandwidth. For $\mathrm{NaCl}: \mathrm{OH}^{-}$, the gain emission spectrum is Gaussian in shape, with a bandwidth of $\delta \nu=45 \mathrm{THz}$ centered at $\nu_{\mathrm{o}}=187.5 \mathrm{THz}(1.60 \mu \mathrm{m})$ [11]. Given these parameters, the normalized gain versus frequency can be expressed as

$$
G(\nu)=\exp \left[-4 \ln 2\left(\nu-\nu_{\mathrm{o}}\right)^{2} / \delta \nu^{2}\right] .
$$

The spectral filtering of the BTP and gain are applied to the main cavity pulse in the frequency domain by taking the product of the pulse spectrum with both filter responses. Expressing the input pulse to this process as $p(t)$, the filtered output becomes

$$
\begin{aligned}
\hat{F}(p(t))= & \frac{1}{2 \pi} \int_{-\infty}^{\infty} \exp (\mathrm{i} \omega t) I_{\mathrm{T}}\left(\frac{2 \pi c}{\omega}\right) G\left(\frac{\omega}{2 \pi}\right) \\
& \times\left[\int_{-\infty}^{\infty} \exp (-\mathrm{i} \omega t) p(t) \mathrm{d} t\right] \mathrm{d} \omega .
\end{aligned}
$$

The effect of the saturable gain can be treated in the time domain [12]:

$$
G=I_{\text {out }} / I_{\text {in }}=\exp \left(\frac{g_{0}}{1+U_{\text {in }} / U_{\text {sat }}}\right),
$$

where $g_{0}$ is the small-signal gain,

$$
g_{0}=\exp \left(\sigma \Delta N_{0}\right)
$$

given $\sigma=9 \times 10^{-17} \mathrm{~cm}^{2}$, the gain cross-section for $\mathrm{NaCl}: \mathrm{OH}^{-}$, and unsaturated population inversion $\Delta N_{0}$, which is proportional to the pumping. $U_{\text {in }}$ and $U_{\text {sat }}$ are input pulse and saturation energy fluences, defined as

$$
\begin{gathered}
U_{\text {in }}(t)=\int_{-\infty}^{\infty} I_{\text {in }}(t) \mathrm{d} t, \\
U_{\text {sat }}=\frac{\hbar \omega}{\sigma} .
\end{gathered}
$$

For $\mathrm{NaCl}: \mathrm{OH}^{-}$laser centered at $\lambda=1.60 \mu \mathrm{m}$, $U_{\text {sat }} \approx 1.38 \mathrm{~mJ} / \mathrm{cm}^{2}$. With these definitions, Eq. (7) expresses the saturable gain as a function of intracavity pulse energy, independent of gain lineshape.

The overall relationship between the fields $a_{1}$ and $b_{1}$ can then be achieved by first filtering $b_{1}$ according to Eq. (6), then multiplying by the onepass gain in Eq. (7) twice, then filtering according to Eq. (6) a final time.

In the external cavity, the fields $a_{2}$ and $b_{2}$ are related by propagation through the single mode 
optical fiber in the external cavity. Given the fiber core radius $\approx 5 \mu \mathrm{m}$ and the duration of typical APM laser pulses $(\approx 100 \mathrm{fs})$, peak pulse intensities within the fiber can exceed $1 \mathrm{GW} / \mathrm{cm}^{2}$. With such high intensities, the fiber exhibits an intensitydependent index of refraction:

$$
n(I)=n_{0}+n_{2} I .
$$

This index variation causes the high-intensity center of the pulse to travel more slowly than the leading and trailing edges, thereby causing compression (frequency upshift) of the optical carrier wave on the trailing edge, and stretching (frequency downshift) on the leading edge. This effect is known as self-phase modulation (SPM), and is usually expressed in terms of the additional phase a pulse acquires in traveling a certain length $L$ of fiber,

$$
\exp (-\mathrm{i} \delta \phi)=\exp \left[-\mathrm{i}\left(\frac{2 \pi L}{\lambda_{0}}\right) n_{2} I(t)\right]
$$

For fused silica fiber, $n_{2}=3.2 \times 10^{-16} \mathrm{~cm}^{2} / \mathrm{W}$, and a length on the order of $20 \mathrm{~cm}$ can typically generate $\pi$ radians of phase across a single pulse.

To express the effects of SPM in this model, we can write $a_{2}$ in terms of $b_{2}$ as

$$
\begin{aligned}
& a_{2}(t)=R_{\mathrm{BS} \gamma} \sqrt{\gamma_{2}} b_{2}(t) \\
& \times \exp \left[-\mathrm{i}\left(\Phi_{0}+\frac{2 \pi L n_{2} \gamma\left(1+\gamma_{2}\right) R_{\mathrm{BS}}\left|b_{2}(t)\right|^{2}}{2 \eta_{0} \lambda_{0}}\right)\right],
\end{aligned}
$$

where $R_{\mathrm{BS}}$ is the intensity reflectivity of the beam-splitter, $\gamma$ is the forward fiber coupling efficiency (between cavity and fiber modes), $\gamma_{2}$ is the back-coupling efficiency (the fraction of the intensity emerging from one pass through the fiber able to be coupled back onto the fiber after retroreflection), and $\eta_{0}$ is the characteristic impedance of air, about $377 \Omega$, used to convert between field strength and intensity. The square-root dependence on $\gamma_{2}$ in the above expression results from taking the back-coupling loss only once, since none of the light is lost exiting the fiber to strike the retroreflector.
Typically, $R_{\mathrm{BS}}=0.5, \quad L \approx 20-50 \mathrm{~cm}, \gamma_{1} \approx 0.5-$ $0.8, \gamma_{2} \approx 0.6-0.95$, and $\Phi_{0} \approx 3.1$ for stable APM operation.

\section{SIMULATION RESULTS}

We first tested the APM model by simulating the steady-state operation of the laser. Given Eqs. (2) and (3) which explain the recombination of pulses $a_{1}$ and $a_{2}$, and Eqs. (6), (7), and (13) which describe the transformation of fields $b_{1}$ and $b_{2}$ by the main and external cavities, the model can be iterated to generate successive $a$ and $b$ fields. With hundreds of round trips through the laser, steady-state is achieved for the typical range of parameters given above.

Table I shows a set of typical laboratory APM laser specifications used as a basis for our model. Using an initial $100 \mathrm{fs}$ Gaussian input pulse in the model, several hundred round trips of the simulator allowed transients to die out. The resulting steady-state pulse and its spectrum after about 500 round-trips are shown in Fig. 3, indicating a $105 \mathrm{fs}$, transform-limited Gaussian pulse train. The output energy of each pulse is calculated to be $6.91 \mathrm{~nJ} / \mathrm{cm}^{2}$,

TABLE I Typical NaCl APM laser specifications

\begin{tabular}{lc}
\hline Average output power & $100 \mathrm{~mW}$ \\
Output beam radius & $2.45 \mathrm{~mm}$ \\
Pulse repetition rate & $80 \mathrm{MHz}$ \\
Pulse width (FWHM) & $100 \mathrm{fs}$ \\
Pulse bandwidth & $4.5 \mathrm{THz}$ \\
$\lambda_{0}$ & $1.60 \mu \mathrm{m}$ \\
\hline
\end{tabular}
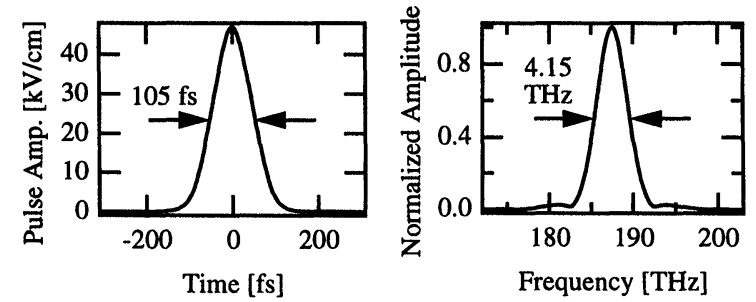

FIGURE 3 Steady-state output pulsewidth and spectrum of the APM model for a typical set of parameters. 
which corresponds to $104 \mathrm{~mW}$ given the pulse width and output beam radius. The steady-state output of the model is thus in excellent agreement with the laser specifications of Table I.

Having verified the accuracy of the APM model in the steady-state, we next varied certain laser parameters, attempting to find a regime of more complicated APM dynamics. With a fixed smallsignal gain and forward and backward fiber coupling coefficients of 0.5 and 0.6 , respectively, we first varied the length of the fiber from $10 \mathrm{~cm}$ to over $50 \mathrm{~cm}$, since the fiber length is the most direct perturbation to the SPM nonlinearity (see Eq. (13)). In varying a model parameter, we iterated the model several hundred times after each parameter adjustment, to insure that the system had settled into a new steady-state. Plotting about 30 successive output pulse energies for each separate fiber length value, Fig. 4 shows that the laser exhibits seemingly unstable behavior after a length of $27 \mathrm{~cm}$.

Figure 5 shows the time progression and phasespace orbit of the output energies, with the discrete data connected for clarity. We observe that the output is periodic, with a period of about 17.5 iterations. Since the laser output itself is periodic (a pulse train with a frequency of $80 \mathrm{MHz}$ ), we can conclude that this behavior is quasiperiodic, since the secondary oscillation period is incommensurate with the fundamental period. The phase-space plot, or first return map ( $n$th versus the $(n+1)$ th iteration) provides a similar conclusion since the orbit becomes continuous as $n \rightarrow \infty$ : no two output

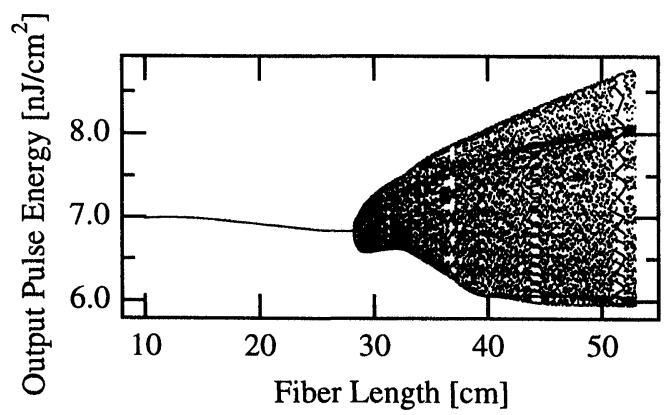

FIGURE 4 Fiber length bifurcation diagram for moderate fiber coupling $\left(\gamma_{1}=0.5\right.$ and $\left.\gamma_{2}=0.6\right)$ showing a large unstable region.
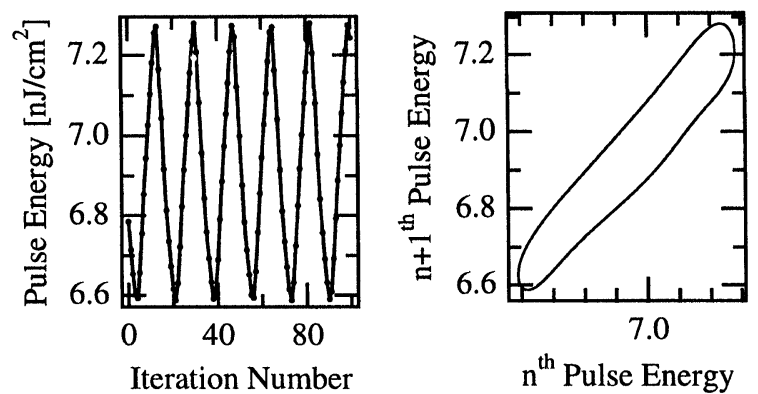

FIGURE 5 Time series and first return map for the unstable region of Fig. 4, demonstrating quasiperiodic output.

pulses are coincident in phase-space, indicating two, incommensurate frequencies. As the fiber length increases beyond $27 \mathrm{~cm}$, similar analysis shows that the output remains quasiperiodic, with the orbit in phase-space simply becoming more convoluted.

We next repeated the fiber length variation with increased forward and backward fiber coupling coefficients of 0.8 and 0.9 , respectively, close to the upper limit of achievable laboratory conditions. We chose to vary the coupling coefficients because they not only perturb the SPM, but also increase the interference between the main and external cavities while remaining readily adjustable laboratory parameters. Figure 6 shows the resulting bifurcation diagram when the output pulse energies are again plotted for each value of fiber length. We see the laser bifurcate from period-one to periodtwo behavior around $9 \mathrm{~cm}$, followed by a perioddoubling cascade into chaos, much like the well-known logistic map. Figure 7 shows a closeup of the unstable region, where the chaotic behavior suddenly drops into a stable orbit near $l=16.30 \mathrm{~cm}$, again period-doubles into chaos, transitions to another seemingly chaotic region, then reverse bifurcates to a stable orbit.

To properly conclude that these instabilities are indeed chaos, we sought to calculate a largest Lyapunov exponent for each of the three unstable regions of Fig. $7: l \approx 15.9-16.3 \mathrm{~cm}, l \approx 16.67-$ $16.76 \mathrm{~cm}$, and $l \approx 16.83-17.12 \mathrm{~cm}$. This involved reconstructing the laser output in delayed-coordinate phase-space, then employing the algorithm of 


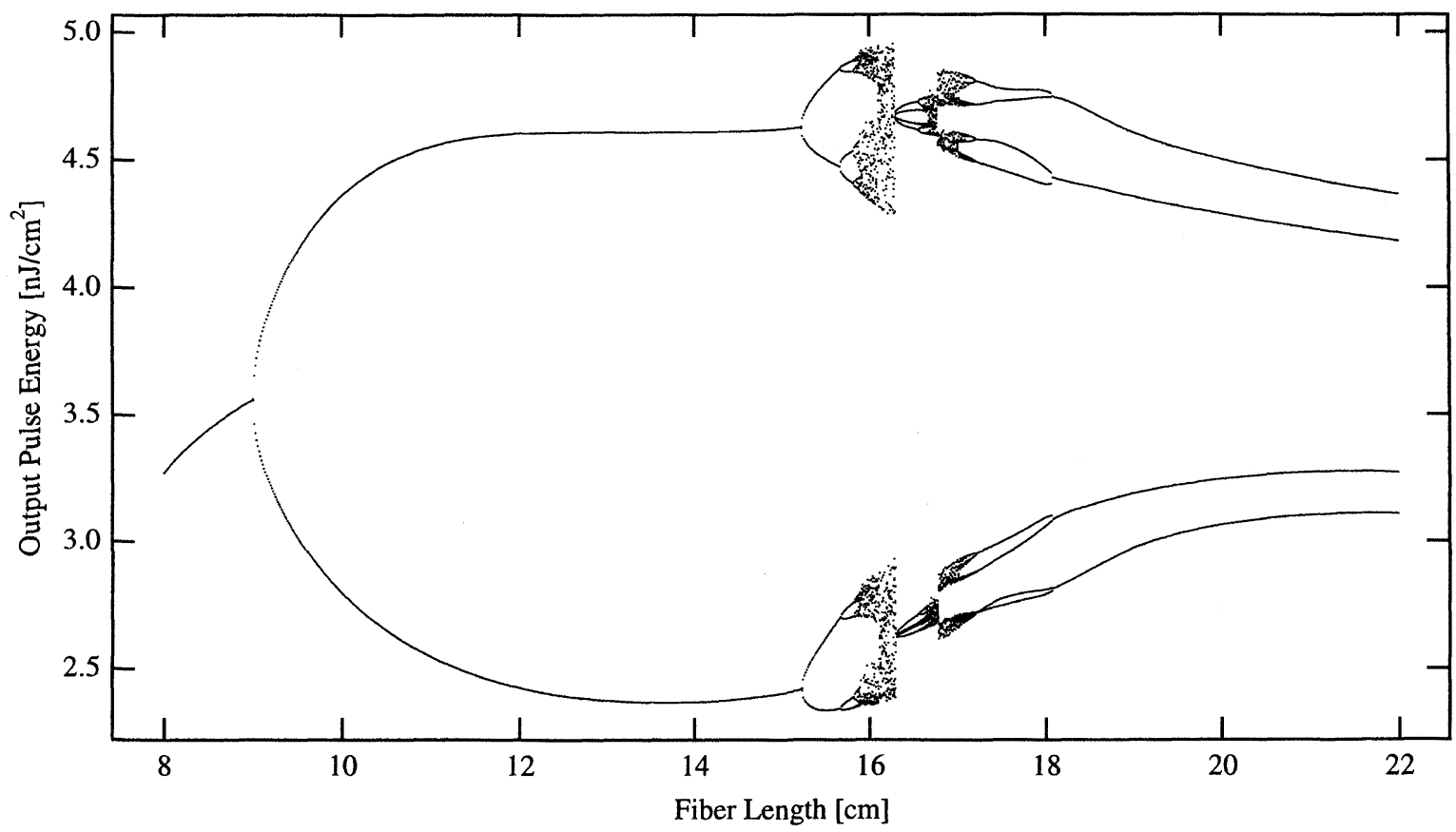

FIGURE 6 Fiber length bifurcation diagram for large fiber coupling $\left(\gamma_{1}=0.8\right.$ and $\left.\gamma_{2}=0.9\right)$ revealing period doubling and chaos.

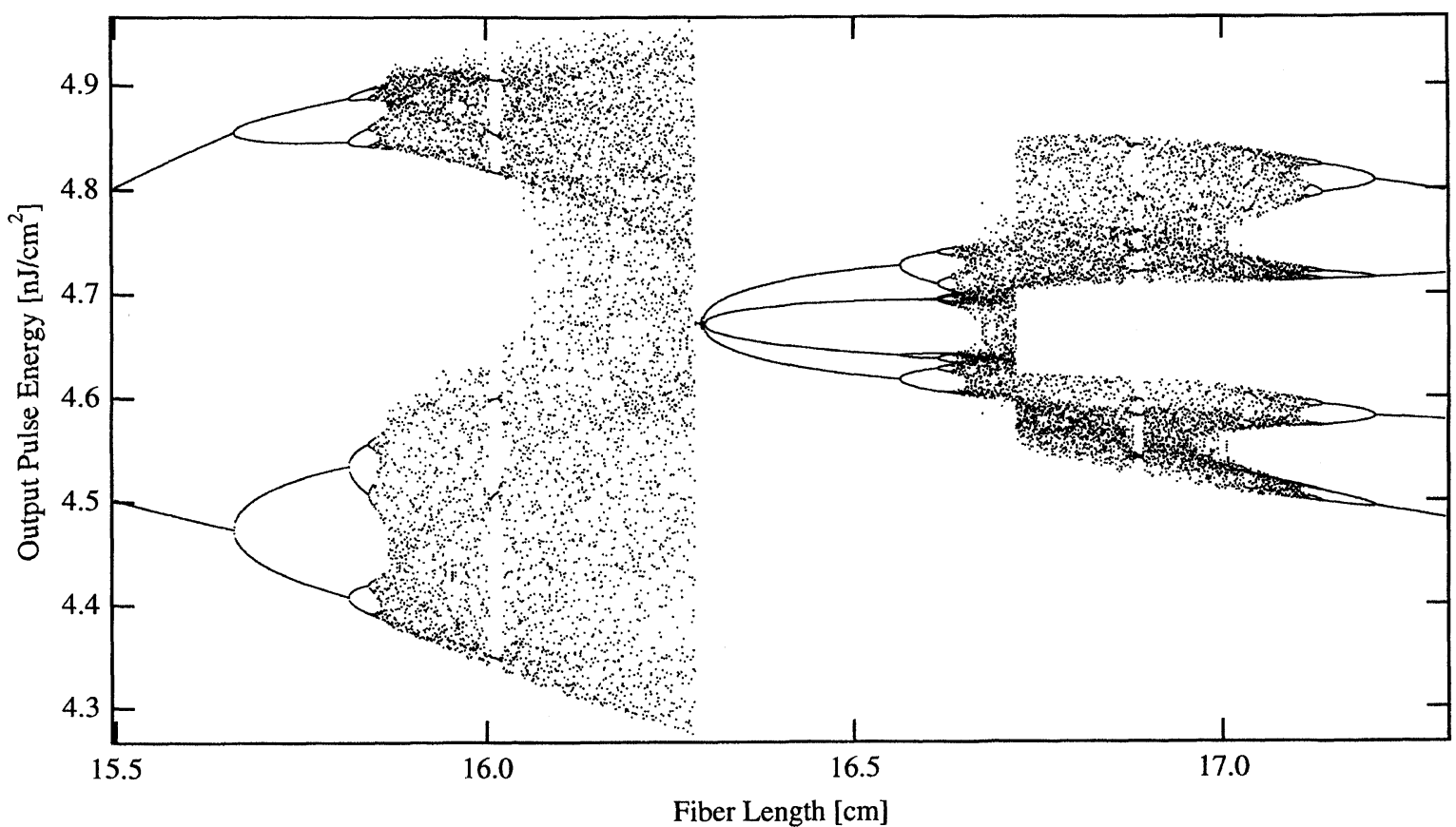

FIGURE 7 Close-up of period-doubling and chaotic regions of the fiber length bifurcation diagram of Fig. 6. 
Wolf et al. [13] to calculate the exponent. Phasespace reconstruction first requires some knowledge of the dimensionality of the APM laser, which is readily provided by the algorithm of Kennel et al. involving false nearest neighbors [14]. Essentially, this method determines the percent of false-nearest neighbors when the laser output is reconstructed in successive integer dimensions, starting from 1. High-dimension systems reconstructed in lowerdimensions will exhibit large percentages of false neighbors, but this percentage will drop to zero when the proper embedding dimension is reached. Figure 8 shows the result of such a calculation for each unstable region of Fig. 7, and the proper embedding dimension is three in each case.

Knowing the proper embedding dimension, we next reconstructed the phase-space orbits, using $n=32000$ output iterations of the model for a given set of parameters in each chaotic region, and the delayed coordinates $n, n+1$, and $n+2$. One attractor from each region is shown in Figs. 9-11: the first two regions actually yield two attractors each, one for each of the two major branches of the bifurcation diagram, while the third region consists of four distinct attractors, due to the underlying period-four behavior. From a graphical viewpoint, the orbits indeed exhibit the qualities of strange attractors, including low topological dimension and folding behavior, and self-similar structure.

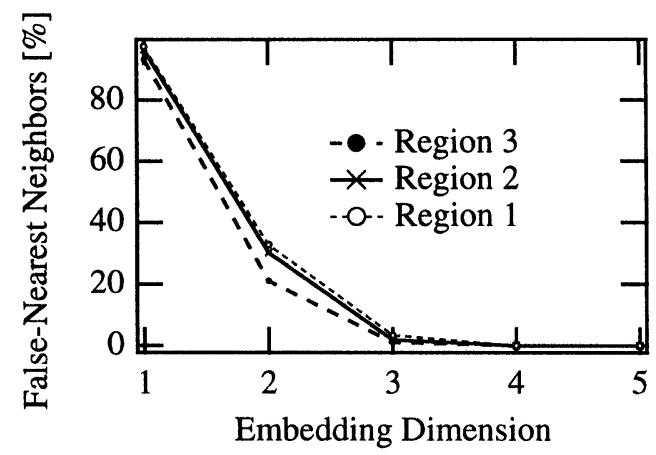

FIGURE 8 False-nearest neighbor embedding dimension calculation for the three prominent regions of instability in Fig. 7.
The best evidence of chaotic behavior however, lies in an estimation of the largest Lyapunov exponent. This exponent was obtained with the Wolf code using a minimum initial displacement of $\sim 10^{-5}$ and a "largeness" condition on the final displacement of $\sim 10^{-2}$ (where the order of the attractor is $\sim 10^{-1}$ ), allowing trajectories to diverge by three orders of magnitude before calculating local exponents. Such a calculation yields $\lambda_{1}=0.24, \lambda_{2}=0.14$, and $\lambda_{3}=0.15 \mathrm{bits} / \mathrm{s}$ for the respective chaotic regions, the positive values indicating the presence of chaos, where nearby trajectories in phase-space locally diverge exponentially.

Figure 7 still raises questions as to the nature of the transitions between the different chaotic regions, or more generally, the routes to chaos in the APM laser. The period-doublings provide an obvious answer as one of the routes into each chaotic region, but the nature of the transitions at $l=16.3 \mathrm{~cm}$ where the chaos suddenly drops into a stable orbit, and $l=16.72 \mathrm{~cm}$ where one chaotic region suddenly jumps to another still remains unknown.

In order to understand these transitions, we consider 16000-iteration reconstructions of the laser output at the critical parameter values. Figure 12 shows the case of $l=16.3 \mathrm{~cm}$, reconstructed three times with successively larger numbers of iterations removed from the beginning of the time series. Plot (a) shows an attractor identical to Fig. 9, from the first chaotic region. Plot (b), with the first 1250 iterations removed, displays a much more "sparse" looking attractor. When the first 2500 iterations are removed, plot (c) shows that the laser is actually exhibiting the stable periodic orbit for $t \rightarrow \infty$. This progression demonstrates what is known as a chaotic transient, where the system follows the nearby, destabilized chaotic attractor for some finite number of cycles, then finally falls onto a different (stable) orbit. Together with the facts that the transition between the chaotic and stable regions is sudden and causes the destruction of the attractor, this chaotic transient provides convincing evidence that the transition at $l=16.3 \mathrm{~cm}$ is a crisis [15]. 


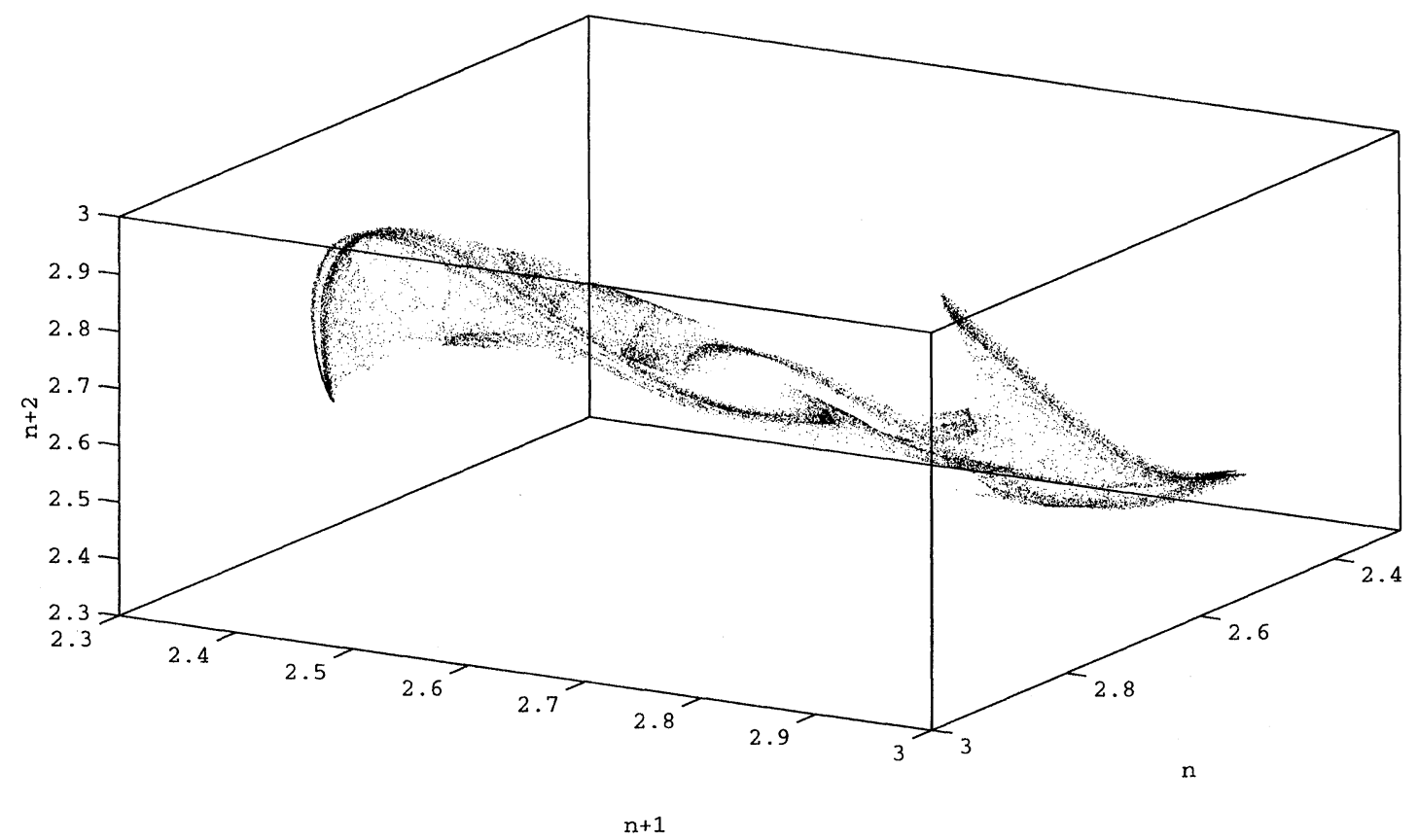

FIGURE 9 Reconstructed chaotic attractor in three dimensions for $l \approx 15.9-16.3 \mathrm{~cm}$.

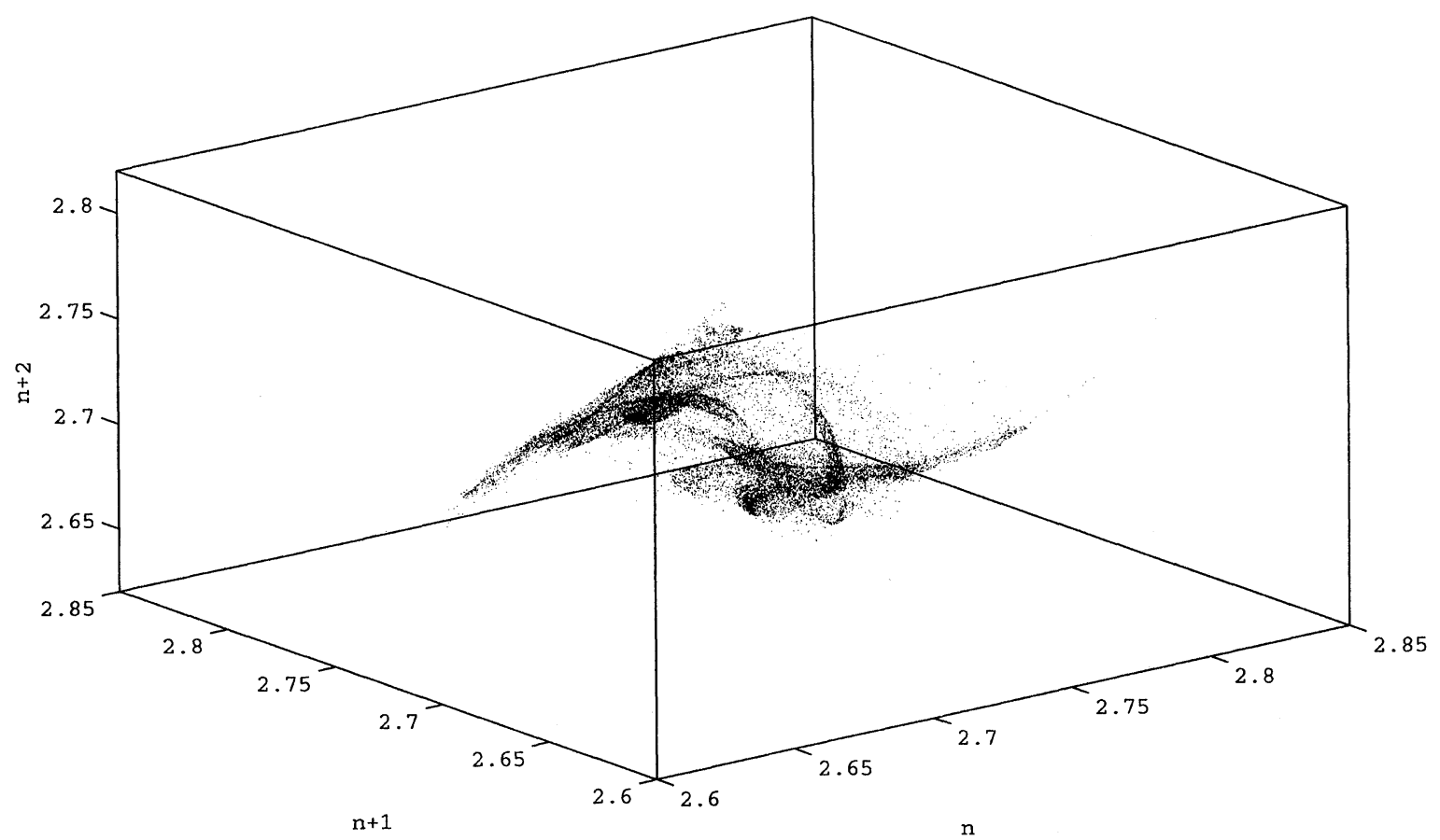

FIGURE 10 Reconstructed chaotic attractor in three dimensions for $l \approx 16.67-16.76 \mathrm{~cm}$. 


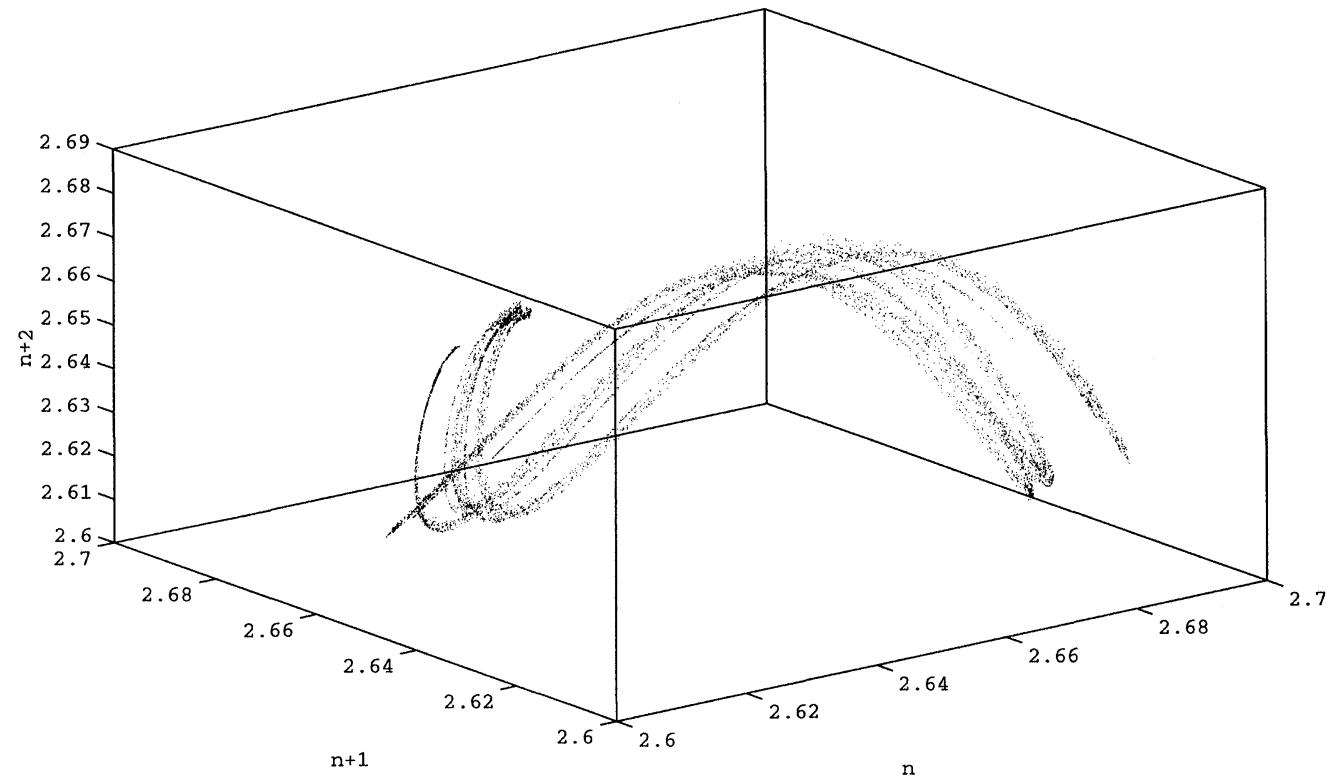

FIGURE 11 Reconstructed chaotic attractor in three dimensions for $l \approx 16.83-17.12 \mathrm{~cm}$.
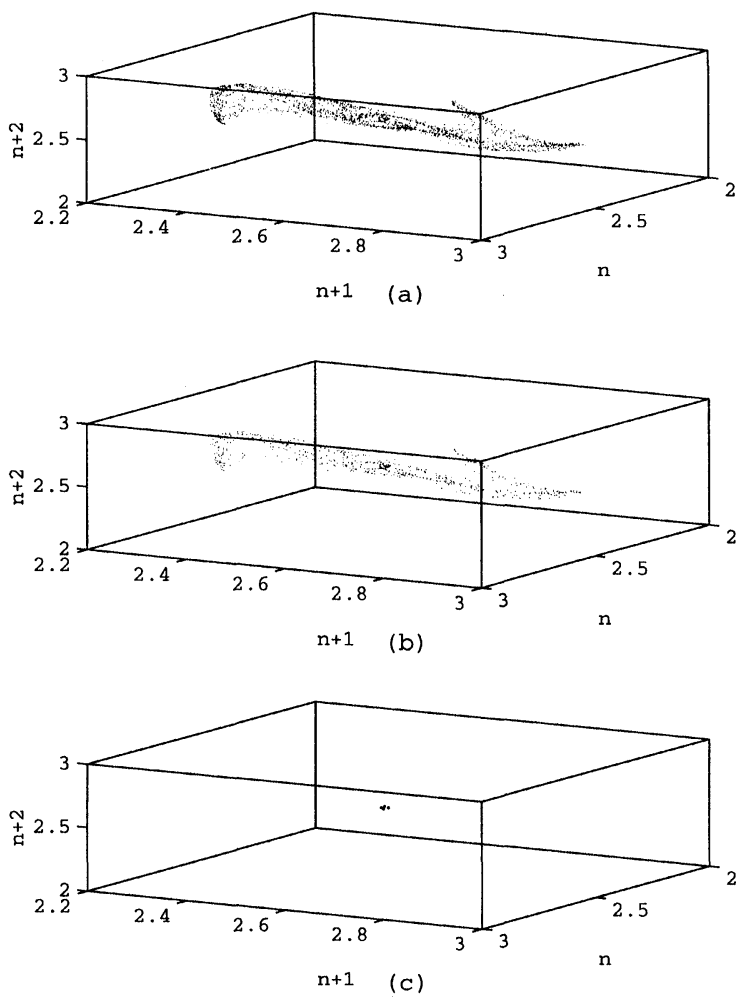

FIGURE 12 Chaotic transient in the APM output near $l=16.3 \mathrm{~cm}$ : (a) shows iterations $1-16000$ containing the large initial chaotic transient preceding a small stable orbit; (b) shows 1251-16000 where a portion of the initial chaotic transient has passed; and (c) shows 2501-16000 where the entire chaotic transient is over, and the system has settled onto the stable orbit. 


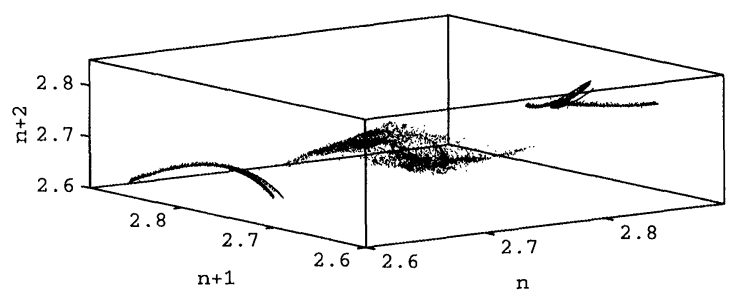

(a)

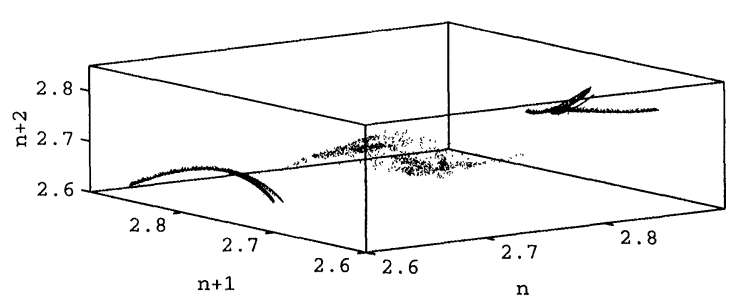

(b)

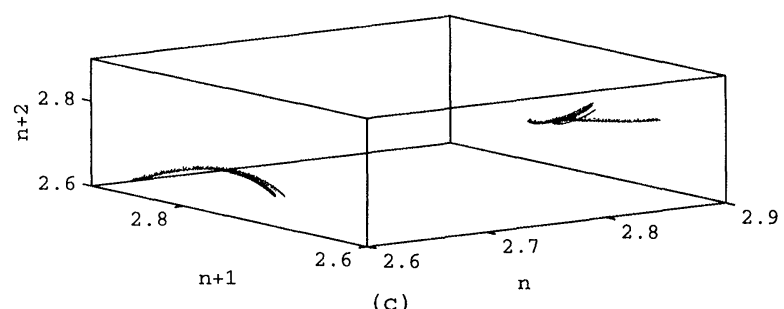

(c)

FIGURE 13 Chaotic transient in the APM output near $l=16.72 \mathrm{~cm}$ : (a) shows iterations $1-12000$ with both an initial chaotic transient and two drastically different coexisting stable chaotic attractors; (b) shows 4001-12000 where a portion of the initial chaotic transient has passed; and (c) shows 8001-12000 where the system has settled onto the two stable coexisting chaotic attractors.

Likewise, we examined the sudden transition at $l=16.72 \mathrm{~cm}$, and found similar behavior. Figure 13 shows the progression of the reconstructed time series, where a region two chaotic transient disappears to leave the region three attractor for $t \rightarrow \infty$. This sudden change of attractor and the presence of the chaotic transient indicate that the region two/three transition is also a crisis.

Since adjusting the fiber couplings had a dramatic effect on the fiber length bifurcation diagram, we decided to investigate fiber coupling as the bifurcation parameter. For a fixed fiber length of $15 \mathrm{~cm}$, we varied the forward and backward fiber coupling coefficients simultaneously $\left(\gamma_{2}=\gamma_{1}+\right.$ 0.1 ). Figure 14 shows the resulting bifurcation diagram, which is very similar to the fiber length case. Aside from variations in the absolute output energy, the only major difference between the fiber coupling and length cases is the unstable region beyond the second crisis at $\gamma_{1}=0.715$. This region exhibits underlying period-two instead of period-four, and also does not reverse bifurcate back to a stable orbit. An embedding dimension calculation shows that this region should be reconstructed in four dimensions, and the resulting largest Lyapunov exponent calculation reveals $\lambda=0.31 \mathrm{bits} / \mathrm{s}$, again indicating chaos.

Beyond fiber parameters, we also varied the small-signal gain (which is proportional to the pumping strength in the laboratory), and found results similar to the fiber length case. For small fiber coupling, the laser exhibited quasiperiodicity for large gain, while higher coupling allowed for period-doubling, chaos, and crises. 


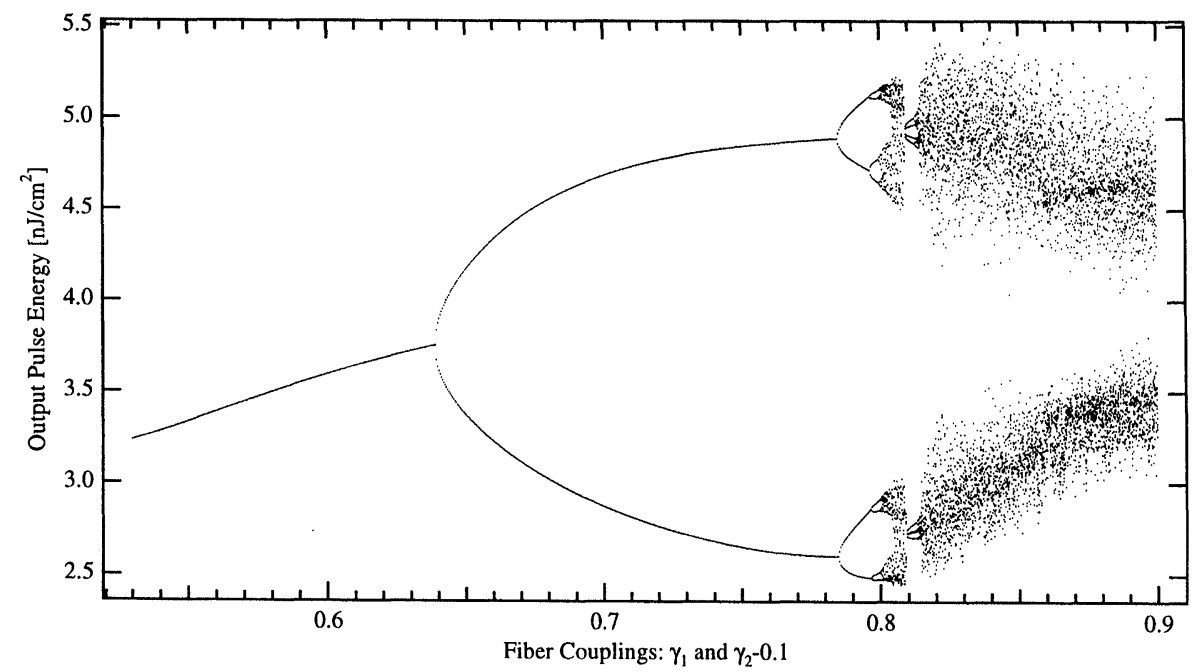

FIGURE 14 Fiber coupling bifurcation diagram, which shows essentially the same features as the fiber length bifurcation diagram.

\section{CONCLUSIONS}

Although the model described above is fairly common for the APM system, one should be aware that certain effects were neglected, including temporal gain saturation (across the pulse profile) and dispersion. The model remains valid in a practical sense however, since temporal gain saturation cannot easily be observed in the laboratory (short timescale), and generally only causes asymmetry in the pulse shape. Moreover, the $\mathrm{NaCl}$ laser is usually operated with dispersion-shifted fiber $(D \approx 0$ at $\lambda=1.55 \mu \mathrm{m})$ and dispersion can be neglected to first order.

More important to the implementation of this model were errors due to overly large bandwidth. Because some of the external cavity energy recirculates through the external cavity without being filtered by the gain and tuner plate in the main cavity, it is possible under conditions of large nonlinearity (long fiber, high coupling, large gain, etc.) for the recirculating pulses to obtain huge amounts of chirp after many iterations, corresponding to pulse bandwidths exceeding $100 \mathrm{THz}$. In the time domain, this corresponds to oscillations on the order of the optical carrier frequency
(187.5 THz), at which point the distinction between the optical wave and its envelope is meaningless; i.e., the situation is non-physical.

When such a situation occurs in the model, the center of the pulses typically becomes extremely narrow, eventually collapsing to a point discontinuity. This event results in a drastically altered pulse integral (energy), and manifests itself in a discontinuity in the bifurcation diagram. In fact, such a non-physical discontinuity in a similar model was erroneously reported as hysteresis [1]. Experimentally, this problem would never arise, because optical components would limit the pulse bandwidth to some finite level. We chose to correct this problem by inserting into the external cavity a filter which approximates the finite-bandwidth coating of a typical laboratory mirror, about $100 \mathrm{~nm}$.

\section{SUMMARY}

We have modeled the APM laser with a simple set of four difference equations which provide a great amount of insight into the complex dynamics of the APM system. In particular, the model verified 
quasiperiodicity, period-doubling, and chaos in the laser output. In addition, we explored the nature of the APM chaos, including the dimensionality of the laser dynamics, the reconstructed chaotic attractors, largest Lyapunov exponents, and crisis transitions between different chaotic regions. Our exploration of several different parameter variations should also prove useful to the development of laboratory experiments to verify and exploit the dynamics of the APM system.

\section{Acknowledgements}

We would like to thank A. Gavrielides, V. Kovanis, and T. Newell of Phillips Laboratory, Kirtland AFB in Albuquerque NM for useful discussion and analysis code. This work was funded by the Joint Service Electronics Program, contract \#F49620-96$1-0162$.

\section{References}

[1] Sucha, G., Bolton, S.R., Weiss, S. and Chemla, D.S. (1996). Period doubling and quasi-periodicity in additive-pulse mode-locked lasers. Optics Letters 20, 1794-1796.

[2] Morgner, U., Rolefs, L. and Mitschke, F. (1996). Dynamic instabilities in an additive-pulse mode-locked $\mathrm{Nd}$ :YAG laser. Optics Letters 21, 1265-1267.

[3] Mollenauer, L.F. and Stolen, R.H. (1984). The soliton laser. Optics Letters 9, 13-15.
[4] Mitschke, F.M. and Mollenauer, L.F. (1986). Stabilizing the soliton laser. IEEE Journal of Quantum Electronics QE-22, 2242-2250.

[5] Mark, J., Liu, L.Y., Hall, K.L., Haus, H.A. and Ippen, E.P. (1989). Femtosecond pulse generation by additive pulse modelocking. IEE Colloquium on 'Applications of Ultrashort Pulses for Optoelectronics' 87, 66.

[6] Ippen, E.P., Haus, H.A. and Liu, L.Y. (1989). Additive pulse mode locking. Journal of the Optical Society of America B 6, 1736-1745.

[7] Yakymyshyn, C.P., Pinto, J.F. and Pollock, C.R. (1989). Additive-pulse mode-locked $\mathrm{NaCl}: \mathrm{OH}^{-}$laser. Optics Letters 14, 621-623.

[8] Sennaroglu, A., Carrig, T.J. and Pollock, C.R. (1992). Femtosecond pulse generation by using an additive-pulse mode-locked chromium-doped forsterite laser operated at 77 K. Optics Letters 17, 1216-1218.

[9] Pinto, J.F., Yakymyshyn, C.P. and Pollock, C.R. (1988). Acousto-optic mode-locked soliton laser. Optics Letters 13, 383-385.

[10] Preuss, D.R. and Gole, J.L. (1980). Three-stage birefringent filter tuning smoothly over the visible region: theoretical treatment and experimental design. Applied Optics 19, 702-710.

[11] Georgiou, E., Pinto, J.F. and Pollock, C.R. (1987). Optical properties and formation of oxygen-perturbed $\mathrm{F}_{2}^{+}$color center in $\mathrm{NaCl}$. Physical Review B 35, 7636-7645.

[12] Haus, H.A. (1975). A theory of forced mode locking. IEEE Journal of Quantum Electronics 11, 323-330.

[13] Wolf, A., Swift, J.B., Swinney, H.L. and Vastano, J.A. (1985). Determining Lyapunov exponents from a time series. Physica D 16, 285-317.

[14] Kennel, M.B., Brown, R. and Abarbanel, H.D. (1992). Determining embedding dimension for phase-space reconstruction using a geometrical construction. Physical Review A 45, 3403-3411.

[15] Grebogi, C., Ott, E. and Yorke, J.A. (1983). Crises, sudden changes in chaotic attractors, and transient chaos. Physica D 7, 181-200. 


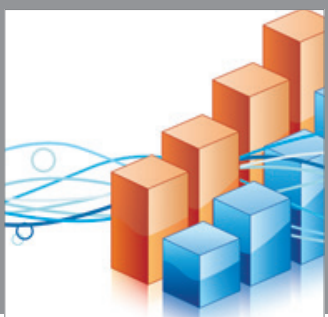

Advances in

Operations Research

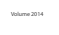

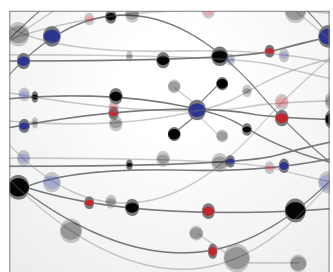

\section{The Scientific} World Journal
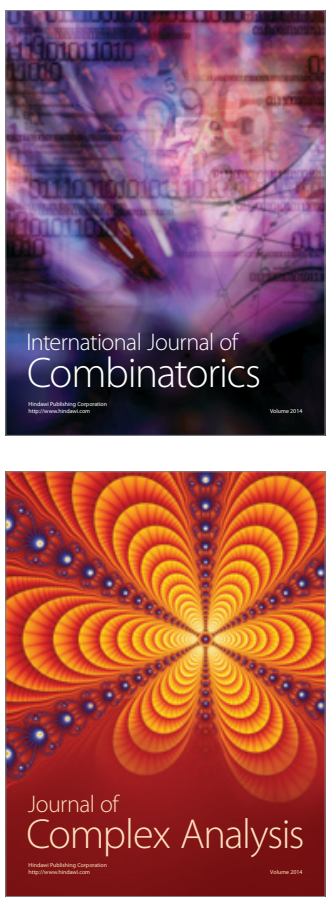

International Journal of

Mathematics and

Mathematical

Sciences
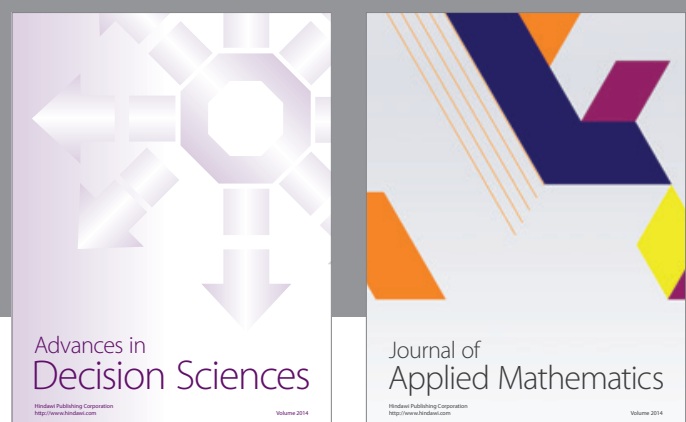

Journal of

Applied Mathematics
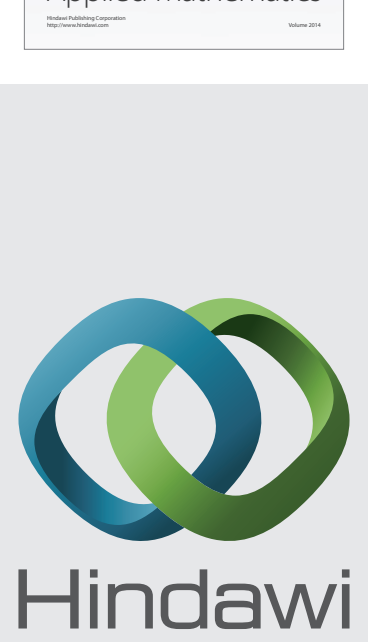

Submit your manuscripts at http://www.hindawi.com
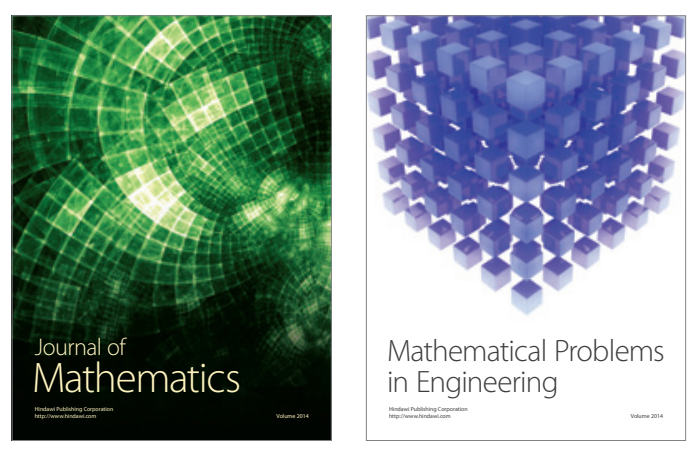

Mathematical Problems in Engineering
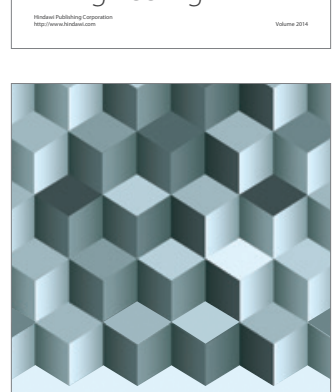

Journal of

Function Spaces
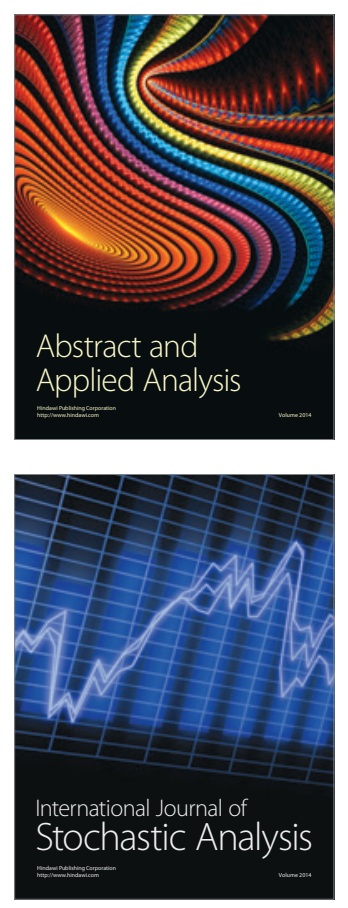

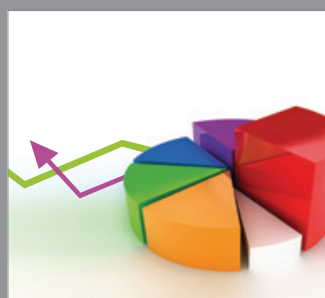

ournal of

Probability and Statistics

Promensencen
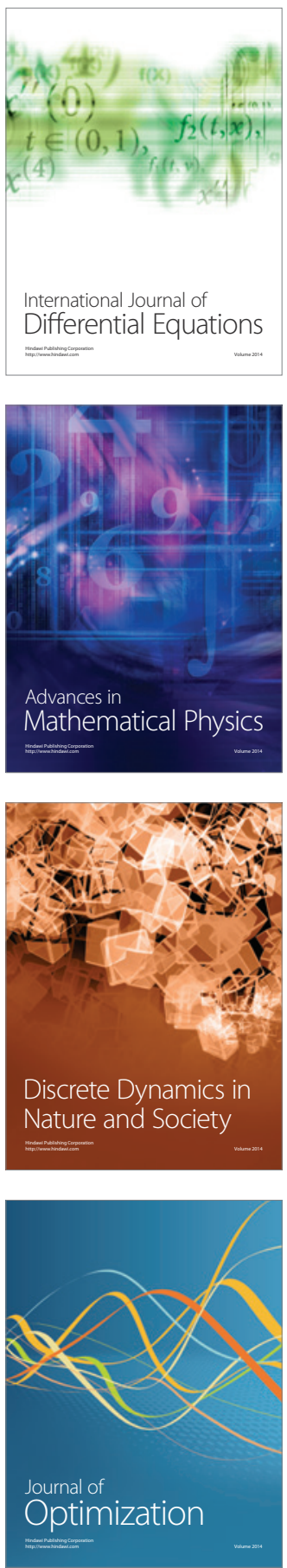